\title{
Scintillating Fiber Devices for Particle Therapy Applications
}

\author{
I. Mattei $^{\circledR}$, G. Battistoni, M. De Simoni, Y. Dong, A. Embriaco, M. Fischetti, V. Giacometti, E. Gioscio, \\ M. Magi, C. Mancini-Terracciano, M. Marafini ${ }^{\circledR}$, R. Mirabelli, S. Muraro, A. Sarti ${ }^{\circledR}$, \\ A. Sciubba, E. Solfaroli Camillocci, M. Toppi, G. Traini, S. M. Valle, and V. Patera ${ }^{\mathbb{D}}$
}

\begin{abstract}
Particle therapy (PT) is a radiation therapy technique in which solid tumors are treated with charged ions and exploits the achievable highly localized dose delivery, allowing to spare healthy tissues and organs at risk. The development of a range monitoring technique to be used online, during the treatment, capable to reach millimetric precision is considered one of the important steps toward an optimization of the PT efficacy and of the treatment quality. To this aim, charged secondary particles produced in the nuclear interactions between the beam particles and the patient tissues can be exploited. Besides charged secondaries, neutrons are also produced in nuclear interactions. The secondary neutron component might cause an undesired and not negligible dose deposition far away
\end{abstract}

Manuscript received May 25, 2018; accepted May 29, 2018. Date of publication June 1, 2018; date of current version August 15, 2018. This work was supported in part by the Centro Nazionale di Adroterapia Oncologica through the Dose Profiler Activity, in part by INFN through the MONDO Project under Young Researchers Grant (2015-2016), and in part by the Scientific Independence of Young Researchers MIUR Funding (2015-2019). (Corresponding author: G. Traini.)

I. Mattei, G. Battistoni, and A. Embriaco are with the INFN Sezione di Milano, 20133 Milano, Italy.

M. De Simoni, C. Mancini-Terracciano, R. Mirabelli, E. Solfaroli Camillocci, and G. Traini are with the Dipartimento di Fisica, Sapienza Università di Roma, 00185 Roma, Italy, and also with the INFN Sezione di Roma, 00185 Roma, Italy (e-mail: giacomo.traini@roma1.infn.it).

Y. Dong and S. M. Valle are with the Dipartimento di Fisica, Università degli Studi di Milano, 20133 Milano, Italy, and also with the INFN Sezione di Milano, 20133 Milano, Italy.

M. Fischetti and M. Magi are with the Dipartimento di Scienze di Base e Applicate per l'Ingegneria, Sapienza Università di Roma, 00185 Roma, Italy, and also with the INFN Sezione di Roma, 00185 Roma, Italy.

V. Giacometti is with the Museo Storico della Fisica e Centro Studi e Ricerche "E. Fermi", 00184 Roma, Italy and also with the Centre for Cancer Research and Cell Biology, Queens University Belfast, Belfast BT7 1NN, U.K.

E. Gioscio is with the Museo Storico della Fisica e Centro Studi e Ricerche "E. Fermi", 00184 Roma, Italy.

M. Marafini is with the Museo Storico della Fisica e Centro Studi e Ricerche "E. Fermi", 00184 Roma, Italy, and also with the INFN Sezione di Roma, 00185 Roma, Italy.

S. Muraro is with the INFN Sezione di Pisa, 56127 Pisa, Italy.

A. Sarti is with the Dipartimento di Scienze di Base e Applicate per l'Ingegneria, Sapienza Università di Roma, 00185 Roma, Italy, also with the INFN Sezione dei Laboratori di Frascati, 00044 Frascati, Italy, and also with the Museo Storico della Fisica e Centro Studi e Ricerche "E. Fermi", 00184 Roma, Italy.

A. Sciubba and V. Patera are with Dipartimento di Scienze di Base e Applicate per 1'Ingegneria, Sapienza Università di Roma, 00185 Roma, Italy, also with the INFN Sezione di Roma, 00185 Roma, Italy, and also with the Museo Storico della Fisica e Centro Studi e Ricerche "E. Fermi", 00184 Roma, Italy.

M. Toppi is with the INFN Sezione dei Laboratori di Frascati, 00044 Frascati, Italy.

Color versions of one or more of the figures in this paper are available online at http://ieeexplore.ieee.org.

Digital Object Identifier 10.1109/TNS.2018.2843179 from the tumor region, enhancing the risk of secondary malignant neoplasms that can develop even years after the treatment. An accurate neutron characterization (flux, energy and emission profile) is, hence, needed for a better evaluation of long-term complications. In this contribution, two tracker detectors, both based on scintillating fibers, are presented. The first one, named dose profiler (DP), is planned to be used as a beam range monitor in PT treatments with heavy ion beams, exploiting the charged secondary fragments production. The DP is currently under development within the Innovative Solutions for In-Beam DosimEtry in Hadrontherapy project. The second one is dedicated to the measurement of the fast and ultrafast neutron component produced in PT treatments, in the framework of the monitor for neutron dose in hadrontherapy project. Results of the first calibration tests performed at the Trento Protontherapy Center and at Centro Nazionale di Adroterapia Oncologica (Italy) are reported, as well as simulation studies.

Index Terms-Charged particle tracker, neutron tracker, particle therapy, range monitoring.

\section{INTRODUCTION}

$\mathbf{P}$ ARTICLE therapy (PT) is presently used to treat many different solid cancers [1], [2]. The main advantage of this technique with respect to the standard radiotherapy is related to the use of heavy charged particle beams, mainly protons and carbon ions, characterized by a high localization of the dose release, helping to better spare the healthy tissues and organs at risk in proximity of the tumor. Carbon ion therapy is especially used to treat radioresistant tumors, due to its high biological effectiveness, increasing the biological damage to tumor cells, with a consequent positive response of the patient to the therapy [3]. The high selectivity of PT in terms of physical and biological doses requires new online beam range monitoring techniques in order to improve the treatment quality assurance and the therapy efficacy. Indeed, possible patient mispositionings and morphological changes with respect to the computed tomography scan could result in a shift of the dose localization from the tumor volume to healthy tissues. All the possible range uncertainty sources [4] are, therefore, taken into account in the treatment plan by the presence of safety margins around the tumor of the order of 1-2 $\mathrm{mm}$ up to $5 \mathrm{~mm}$ or more in the case of ${ }^{12} \mathrm{C}$ ion therapy [5], [6]. The beam range monitoring in PT exploits the secondary radiation produced by the hadron beam interacting with the patient's body: 1) prompt photons [7]-[9] with energies in the range of $\sim 1-10 \mathrm{MeV}$ produced by the nuclear deexcitation of excited nuclei; 2) pairs of back-to-back $511 \mathrm{keV}$ photons [positron emission tomography (PET) photons] produced by the annihilation of positrons coming from 


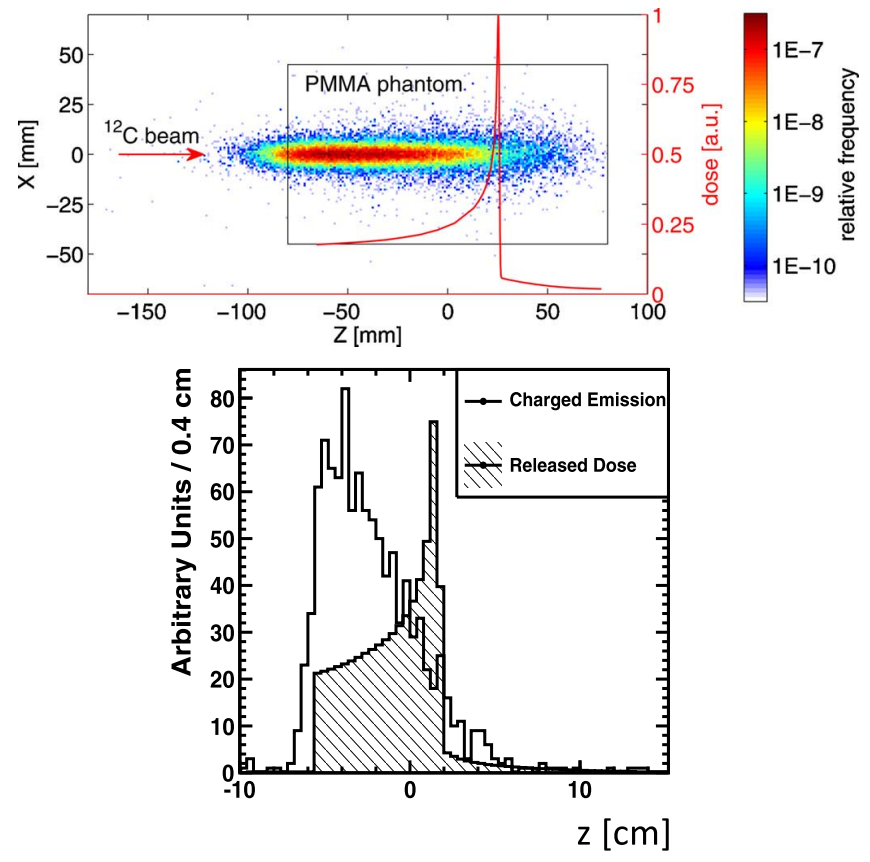

Fig. 1. $x z$ distribution of the charged secondary particles detected at $30^{\circ}$ with respect to the beam incoming direction and emitted by a $250 \mathrm{MeV} / \mathrm{u}{ }^{12} \mathrm{C}$ ion beam impinging on a polymethyl methacrylate (PMMA) target (black rectangle) (top). The relative depth-dose distribution is also shown (red curve) [13]. Longitudinal emission profile of charged secondary particles, detected at $90^{\circ}$ with respect to the beam incoming direction, produced by a $220 \mathrm{MeV} / \mathrm{u}{ }^{12} \mathrm{C}$ ion beam impinging on a PMMA target $\left(5 \times 5 \times 20 \mathrm{~cm}^{3}\right)$ (bottom). The depth-dose distribution (hatched area) is superimposed [12].

$\beta^{+}$emitters [10], [11]; and 3) charged secondary particles [12], [13] produced in nuclear interactions.

In PT, recently, a beam range monitor technique based on the detection of prompt photons has been tested on a patient using the knife-edge slit camera developed by IBA in collaboration with Politecnico di Milano with good results [14], [15]. At the end of 2016, the imaging system based on the in-beam detection of PET photons [16], developed within the Research and Development in Hadrontherapy (RDH)/Innovative Solution for In-Beam DosimEtry in Hadrontherapy (INSIDE) project [17], has been installed at Centro Nazionale di Adroterapia Oncologica (CNAO) in Pavia (Italy) and tested on a patient undergoing a proton therapy treatment with promising results. The beam range monitoring techniques based on the detection of PET photons and prompt photons, when applied to carbon ion beam treatments, do suffer from a large background, difficult to suppress, induced by the ${ }^{12} \mathrm{C}$ ion beam nuclear interactions with tissues.

On the other hand, charged secondary particles are particularly suitable to monitor the beam range during heavy ion beam PT, with carbon, helium, or oxygen ions. The highly penetrating secondary charged fragment component might escape the patient and be detected with very high efficiency in a nearly background-free environment. Recent experiments demonstrated that the longitudinal emission distribution of such fragments is related to the beam range inside a human tissue-equivalent target, as shown in Fig. 1.

Charged secondary fragments are preferentially forward emitted [13] but a non-negligible fraction of secondary protons produced by ${ }^{12} \mathrm{C}$ ion beams are emitted at large angles

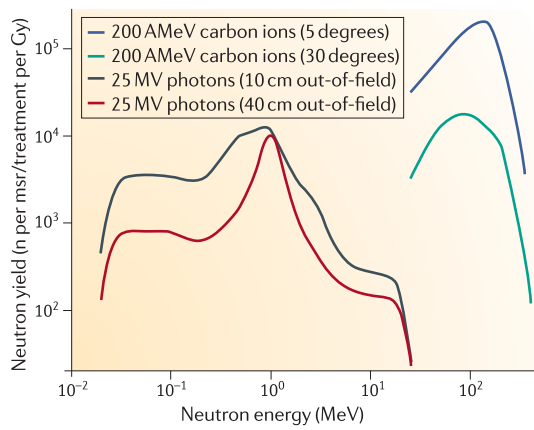

Fig. 2. Secondary neutron yield as a function of the energy of photoneutrons produced by $25 \mathrm{MV}$ X-rays and secondary neutrons produced by nuclear interactions of $220 \mathrm{MeV} / \mathrm{u}$ carbon ions with water target. Photoneutrons were measured at $10 \mathrm{~cm}$ (black) or $40 \mathrm{~cm}$ (red) from the target area. Secondary neutrons produced by ${ }^{12} \mathrm{C}$ ions were measured at $5^{\circ}$ (blue) and $30^{\circ}$ (red) with respect to the beam direction. These different spectra result in different (organ-specific) risk factors [19], [21].

$\left(\theta=60^{\circ}-90^{\circ}\right)$ as well [12]. The production of secondary protons at large angles is particularly interesting due to geometrical consideration: the beam transverse profile worsens the spatial resolution of particles reconstructed trajectories by a factor proportional to $(\sin \theta)^{-1}$. Moreover, going to large angles simplifies the location of the secondary protons detector in a treatment room with respect to the patient positioning [12]. There are two main shortcomings for a beam range monitor based on charged secondary particles: 1) they suffer multiple scattering (MS) interactions when crossing the patient and 2) they need a minimum kinetic energy to exit the patient body; therefore, a limited fraction of produced particles can actually be exploited for monitoring purposes. Such factors limit the spatial resolution that can be achieved when reconstructing the Bragg peak (BP) position, i.e., the beam range. To collect a sufficient statistics of charged secondary particles, the detector solid angle needs to be increased in order to achieve the needed resolution on the BP position. In order to match the requirements set by the online monitoring applications, a detector was designed within the RDH/INSIDE project: the dose profiler (DP) [18], a charged secondary particles tracker based on scintillating fibers. The DP layout and the first calibration test at the Trento Protontherapy Center (Italy) will be presented in Section II.

Together with the development of an online beam range monitoring technique in PT, to improve the accuracy and the quality assurance of the therapy, the secondary neutron component has to be taken into account when planning the treatments. Secondary fast and ultrafast neutrons are produced in nuclear interactions of carbon ion beams with patient tissues (see Fig. 2) as well as charged secondary particles, with the energy of hundreds of megaelectronvolt. They may deposit their energy far from their production point, causing possible secondary-malignant neoplasms especially in pediatric patients who have a longer life expectation [19]. Nowadays, an accurate and complete characterization of secondary neutron energy spectra as a function of the emission angle is still missing: detectors capable of reconstructing the neutrons direction at energies of interest in PT have not yet been developed. 
Therefore, the treatment planning system presently has not an accurate prediction capability of the effects induced by the neutron dose release. The aim of the Monitor for Neutron Dose for Hadrontherapy (MONDO) experiment [20] is to characterize the fast and ultrafast neutron radiation produced in PT treatments, to assess the neutron-induced therapy risk, to optimize the treatment plan, and, possibly, to understand if a correlation exists between the neutron emission and the primary beam range for range monitoring purposes. The MONDO detector is a secondary fast and ultrafast neutrons tracker, using the scintillating fibers as an active medium. Its layout and the first simulation results will be presented in Section III together with the first results obtained at the protontherapy center in Trento.

\section{Dose Profiler}

The proposed detector is being developed in the framework of the INSIDE project which proposes a multimodal inbeam dose monitor including both a PET photons detector and a charged particle tracker. Such a monitor is designed to be integrated in a treatment room of the CNAO center. The DP is a charged secondary particles tracker based on scintillating fibers that will reconstruct the charged secondaries emission profile. A reconstruction software and a back-filtering technique based on a fast Monte Carlo (MC) code, will be used to consider the tracks attenuation due to the length of the path traveled by the secondary proton inside the patient and the density of the different tissues crossed before exiting the target. The reconstructed emission profile will, therefore, be compared with a reference scenario, where the correlation between the profile and the beam range inside the patient has been established.

\section{A. Detector Layout}

The DP is composed by six planes of two orthogonally placed scintillating fiber layers, each made of 384 fibers of $500 \times 500 \mu \mathrm{m}^{2}$ squared section (multicladding BCF-12 from Saint-Gobain), in order to reconstruct the 3-D coordinates of the proton track. Fibers composing a layer are glued together. The scintillating fiber planes are followed by two planes of orthogonally placed plastic scintillator bars. Each bar (EJ-200 from Eljen) is a polyvinyltoluene-based scintillator with low atomic number $($ Zeff $=3.4)$ placed just after the tracker, with dimensions $1.2 \times 19.2 \times 0.6 \mathrm{~cm}^{3}$. The plastic scintillator planes will perform the charged tracks energy measurement to exclude the lowest energy fragments which suffer from larger MS. The minimal separation of $2 \mathrm{~cm}$ between two consecutive planes is a tradeoff between the need to increase the tracker spatial resolution and the detector compactness, important due to the space limitations in a treatment room, and large geometrical acceptance, which increases the reconstruction efficiency. The choice of the fiber size is the result of an optimization aiming to balance signal amplitude and the total amount of material to be crossed by charged particles. The total size of the detector is $28 \times 28 \times 31 \mathrm{~cm}^{3}$. Fig. 3 shows the DP layout and the principle of secondary proton reconstruction.

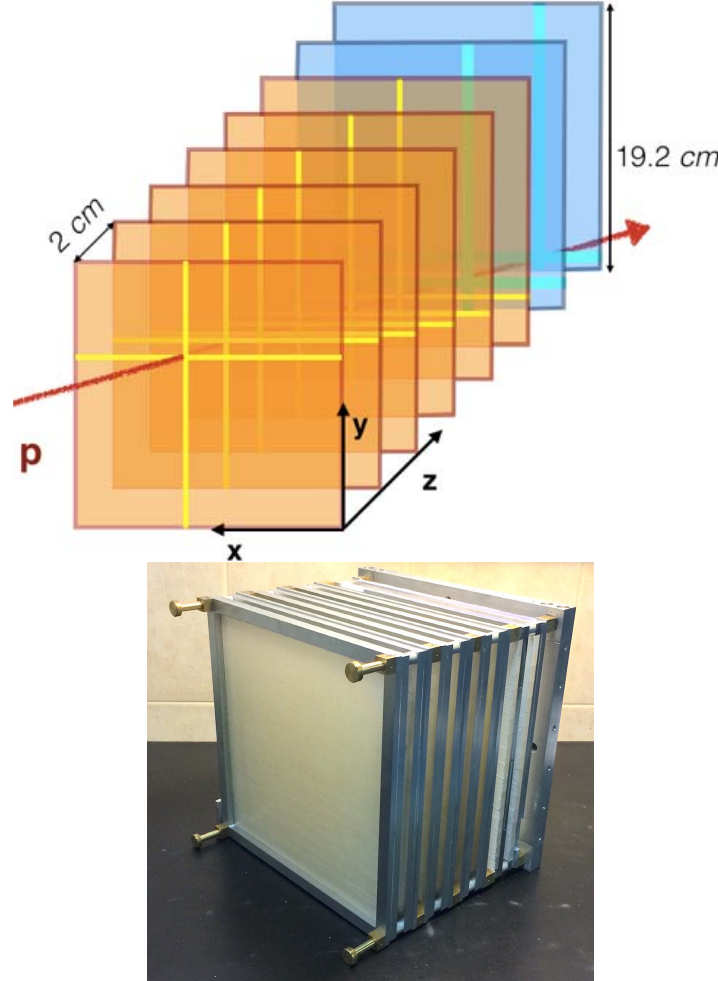

Fig. 3. Scheme of the detector and detection principle for a proton (top). In orange, the six scintillating fiber planes are depicted, while the two plastic scintillator planes are in light blue. Picture of the detector planes in the assembling phase (bottom).

In order to optimize the charged particle detection, the choice of the angle at which the detector has to be placed with respect to the beam direction is crucial. At narrow angles, there is the advantage that the emission flux is enhanced. On the other hand, the detected emission shape is convoluted with the transverse beam spot size projected on the beam line; therefore, the spatial resolution on the emission shape worsens for angles different from $90^{\circ}$. As a compromise, in INSIDE, a detection angle of $60^{\circ}$ has been chosen.

The fibers readout is performed by means of $1 \mathrm{~mm}^{2}$ silicon photomultipliers (SiPMs). Since each fiber is $500 \mu \mathrm{m}$, each SiPM reads two adjacent fibers. In order to read the whole 384 fibers, SiPMs are alternately coupled to the fibers on both sides, so that a single layer is read by 192 channels. The SiPMs are readout by BASIC32_ADC [22], 32-channel Application Specific Integrated Circuits (ASICs) specifically developed to readout SiPM detectors in medical imaging applications. The BASIC32_ADC, 32-channel ASICs are configured and readout by a set of field-programmable gate arrays. The system has been tested at the CNAO center in clinical conditions, and a maximum sustainable rate of $\sim 12000 \mathrm{~s}^{-1}$ has been measured (see Section II-C). To avoid the development of a dedicated read-out subsystem, the front-end board of the tracker is also used to read the plastic scintillator planes. The detector provides an internal trigger system, optimized for proton tracks triggering.

\section{B. Performances Study}

In May 2017, a calibration test has been performed at the Trento Protontherapy Center using proton beams, with 

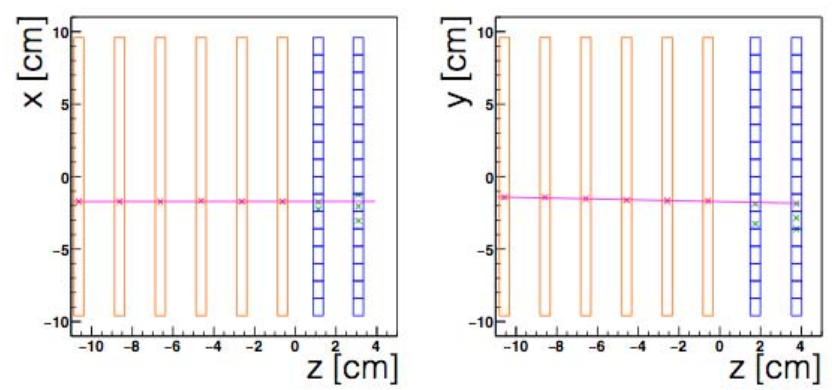

Fig. 4. Example of a reconstructed $70 \mathrm{MeV}$ proton track (magenta line) in the $x z$ (left) and $y z$ (right) views. The single hits shown as red crosses are clusters of fibers, while the green crosses are clusters of scintillating bars. A schematized DP layers geometry with scintillating fiber layers in orange and the plastic scintillator layer in blue is also represented.
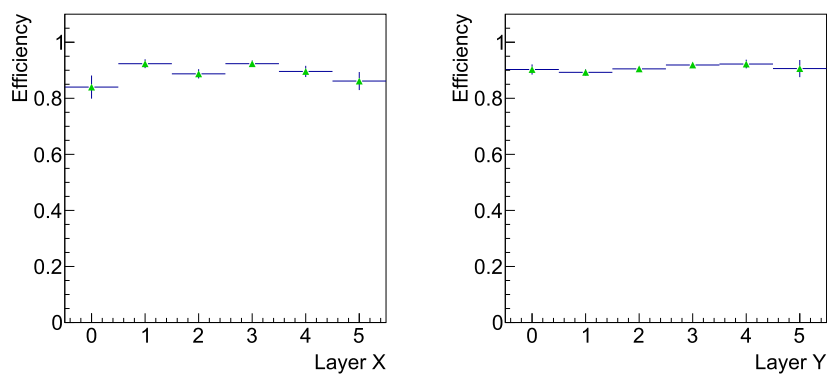

Fig. 5. Single scintillating fibers layer efficiency of the $x z$ (left) and $y z$ (right) views.

energies ranging from 70 to $220 \mathrm{MeV}$ directed along the $z$-axis, impinging directly in the $x y$ DP plane. The beam transversal profile was Gaussian shaped with a sigma $\sigma_{\text {beam }}=3-7 \mathrm{~mm}$ at the isocenter, depending on the energy. An example of a reconstructed proton track in the two separate DP views $(x z$ and $y z)$ is shown in Fig. 4 together with the DP geometry scheme. A track is reconstructed when at least four clusters (one per layer) are present. The single-layer efficiency for the tracker layers (scintillating fiber layers) has been computed from dedicated runs: the DP has been irradiated with a $91 \mathrm{MeV}$ proton beam, and it was shifted along the $x$ and $y$ positions in order to irradiate the whole DP area. The layer efficiency was computed asking for the presence of the probed layer once a track is reconstructed. The measured efficiency is $\sim 90 \%$ for both the $x z$ and $y z$ views, as shown in Fig. 5. The $10 \%$ inefficiency is due to fibers dead space alignment between layers and possible miscoupling of fibers to SiPMs.

The spatial resolution on a single reconstructed proton track has been measured at the isocenter, at $\sim 50 \mathrm{~cm}$ from the DP entrance face. The tracks reconstructed by the DP were extrapolated to the isocenter and the $x y$ distribution is shown in Fig. 6 for the $70 \mathrm{MeV}$ proton beam. The resolution $\sigma_{\text {meas }}$ on a single view and on a single reconstructed track has been measured fitting with a Gaussian function, the $x$ (or $y$ ) projection of the 2-D distribution. Knowing the nominal value of $\sigma_{\text {beam }}$ at each beam energy, the spatial resolution of the DP has been computed and values between 3-5 $\mathrm{mm}$ have been obtained on a single reconstructed track.

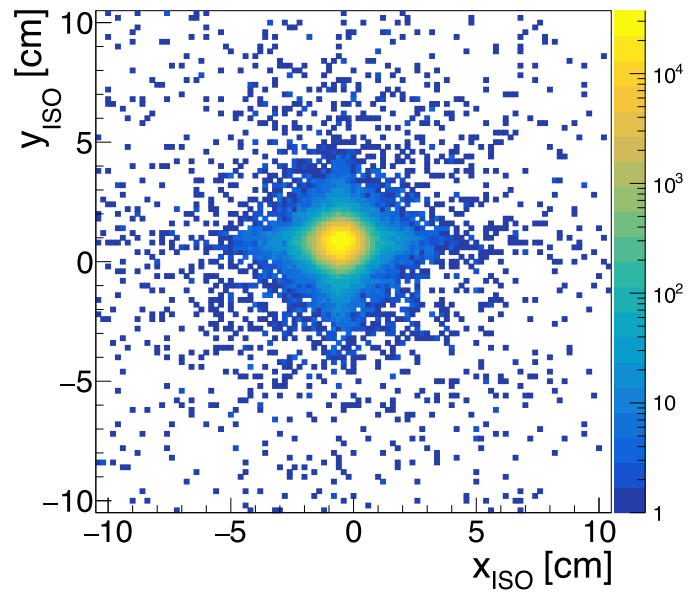

Fig. 6. Bidimensional distribution of the reconstructed $x$ - and $y$-coordinates of the proton tracks reconstructed by the DP and extrapolated at the isocenter for the $70 \mathrm{MeV}$ proton beam case.

\section{Test of DAQ in Clinical Condition}

In July 2017, a test of the data acquisition (DAQ) of the DP at the CNAO treatment center has been performed in order to verify the operation of the DP detector in clinical conditions. Carbon ion beams of several energies impinged on an anthropomorphic head phantom (the Alderson RANDO® phantom) in treatmentlike conditions and the charged secondary particles exiting RANDO were collected by the DP placed at $90^{\circ}$ or $60^{\circ}$ with respect to the beam direction. The beam was provided with a treatment-like intensity of the order of $10^{7}-10^{8}$ ions/s. The measured DP mean rate was between $6000 \mathrm{~s}^{-1}$ and $8000 \mathrm{~s}^{-1}$, with a maximum sustainable rate of $\sim 12000 \mathrm{~s}^{-1}$, and a $80 \mu \mathrm{s}$ dead time. Such rate was due to a preliminary coding of the data transfer of the device that resulted in a huge dead time. Currently, the data transfer has been optimized, reaching a design rate $>100000 \mathrm{~s}^{-1}$ that will be verified in further DAQ tests at CNAO. Considering the obtained results, the DP will not be used to monitor the range of the single pencil beam (PB) irradiating each tumor voxel, but the current monitoring target is a tumor slice or few PB irradiating the same tumor area.

\section{MONDO DETECTOR}

The aim of the MONDO detector is to track secondary neutrons produced in nuclear interactions of PT heavy ion beam with patient tissues, reconstructing the neutron 4-momentum exploiting the double elastic scattering (DES) in the tracker interacting material (see Fig. 7).

In a single elastic scattering, a recoil proton is produced and the following equation describes the relationship between the neutron $\left(\vec{p}_{n}\right)$ and proton $\left(\vec{p}_{p}\right)$ momenta and the proton diffusion angle $\left(\theta_{p}\right)$ :

$$
\vec{p}_{n}=\frac{p_{p}}{\cos ^{2} \theta_{p}} \hat{n}
$$

If the diffused neutron ( $\left.n^{\prime}\right)$ undergoes another elastic scattering, and another recoiling proton $\left(p^{\prime}\right)$ is produced, using 


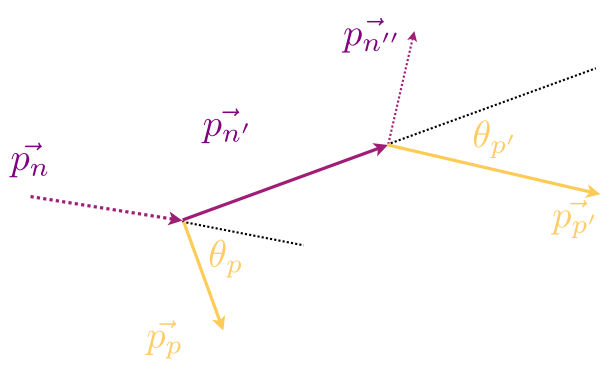

Fig. 7. Scheme of a DES reaction.

the same relation in (1), and knowing the diffused neutron direction (and therefore, the diffused $p^{\prime}$ angle $\theta_{p^{\prime}}$ ) connecting the two interaction vertexes, it is possible to reconstruct the primary neutron momentum and incoming direction.

In order to track neutrons and to measure their 4-momentum, the MONDO detector will, therefore, track the two recoiling protons from neutron DES reactions.

\section{A. Detector Layout}

To maximize the neutron DES interaction probability, the MONDO detector will be made of a highly hydrogenated material as an active medium. It will be composed by subsequent orthogonal layers of $250 \times 250 \mu \mathrm{m}^{2}$ squared plastic scintillating fibers that will contemporarily act as a target for neutrons and detector for recoil protons. A proton crossing layers of fibers will lose energy, producing scintillation photons that will be detected and allowing to reconstruct the proton direction. The proton energy will be measured from its range in the tracker material, asking for the full containment of the recoiling track. The small fiber size is, therefore, a tradeoff between the scintillation light that needs to be detected and the achievable spatial resolution on the reconstructed track. Three is the minimal number of crossed layers in both orthogonal views in order to reconstruct a proton track, corresponding to a total amount of plastic scintillating material of $1.5 \mathrm{~mm}$ and to a proton energy threshold of $12 \mathrm{MeV}$. The final detector size will be $10 \times 10 \times 20 \mathrm{~cm}^{3}$, allowing to measure neutrons up to $400 \mathrm{MeV}$ of energy.

\section{B. Simulation Results}

An MC simulation using the FLUktuierende KAskade (FLUKA) code [23], [24] has been developed in order to study the detector performances. In Fig. 8, the energy deposited in a single fiber as a function of the proton kinetic energy is shown. Protons with $E_{\text {kin }}>100 \mathrm{MeV}$ deposit an energy of $\sim 100 \mathrm{keV}$. Taking into account the fiber trapping efficiency of $7.2 \%$ and a light yield of $8000 \mathrm{ph} . / \mathrm{MeV}$, as provided by Saint-Gobain, for a deposited energy of $100 \mathrm{keV}, \sim 60$ photons are emitted from exit faces of the fibers.

To compute the detection efficiency $\epsilon$ of the MONDO detector, $5 \times 10^{6}$ neutrons have been generated with a flat energy spectrum in the range of $20-400 \mathrm{MeV}$ in a $5^{\circ}$ aperture cone centered in the tracker entrance face. The detection efficiency, defined as the number of DES events, where the recoil protons are fully contained over the number of neutrons produced in the corresponding energy bin, is shown in Fig. 9. The energy bin width is $30 \mathrm{MeV}$. The plateau level clearly

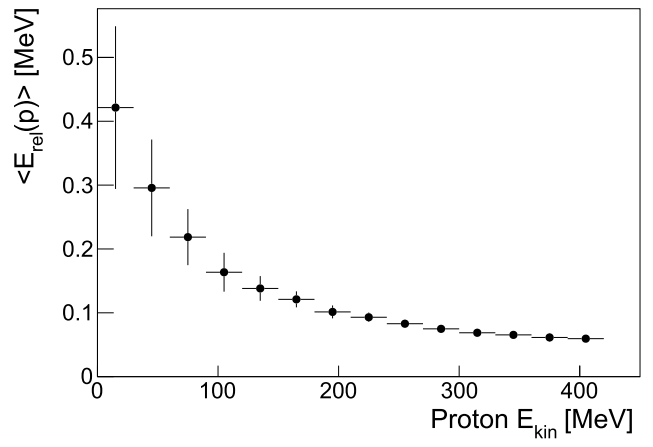

Fig. 8. Mean energy deposited in a single $250 \mu \mathrm{m}$ fiber as a function of the proton kinetic energy [20].

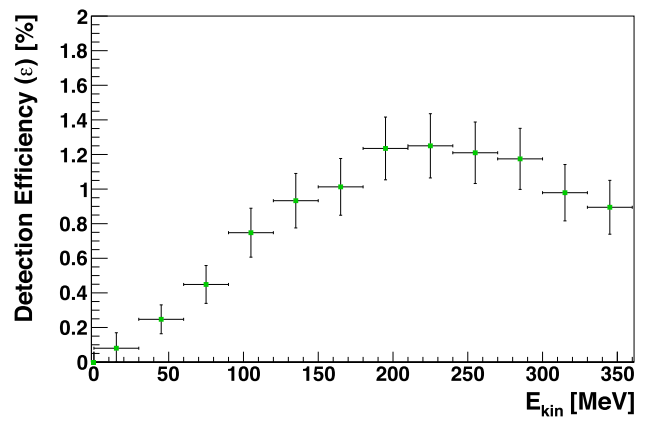

Fig. 9. Detection efficiency as a function of the incoming neutron energy, obtained with an MC simulation [20].

depends on the detector size: the larger the neutron energy, the larger the mean recoil proton energy, the larger should be the detector size to fully contain it.

It is clear that if the kinetic energy of the protons can be measured by removing the full containment constraints, the detector efficiency would improve, especially for high energy.

\section{Calibration Tests}

A first calibration test of a MONDO detector prototype with smaller size, named Penelope, has been performed in 2016 with electron beams at the beam test Facility of Laboratori Nazionali di Frascati (Italy) [26]. In May 2017, a calibration with proton beams has been performed at the Trento Protontherapy Center and here first results are reported.

Penelope $\left(4 \times 4 \times 4.8 \mathrm{~cm}^{3}\right)$ has been assembled to perform preliminary studies on the MONDO detector response. The tracker prototype has been placed along the proton beam line, in correspondence of the isocenter. The proton beam energy was ranging between 70 and $220 \mathrm{MeV}$, with intensities of the order of $\sim 10^{3}$ particles/s, and it was Gaussian shaped, with a 3-7 $\mathrm{mm}$ sigma. A Penelope face has been read-out by the silicon sensor single photon avalanche diode (SPAD)-net [25], a device developed by the Bruno Kessler Foundation (FBK). SPAD-net is based on the SPAD technology and has been used here as a prototype of the Spad Based Acquisition for the Mondo experiment (SBAM) optimized sensor not yet available [26]. SPAD-net is a SPAD matrix of $16 \times 8$ pixels $\left(\sim 600 \times 600 \mu \mathrm{m}^{2}\right)$, for a total active area of $1 \times 0.5 \mathrm{~cm}^{2}$. Therefore, only a small portion of the tracker prototype has 


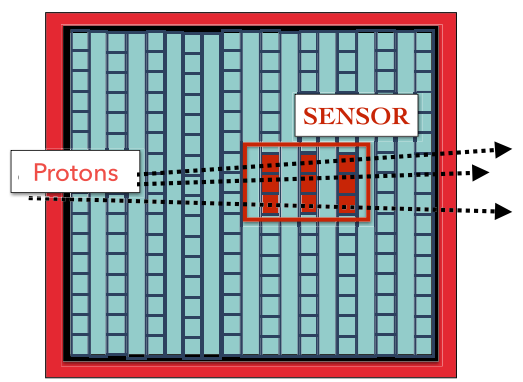

Fig. 10. Top view of the MONDO tracker prototype, Penelope SPAD-net [25], the readout sensor prototype developed by FBK, is also shown.

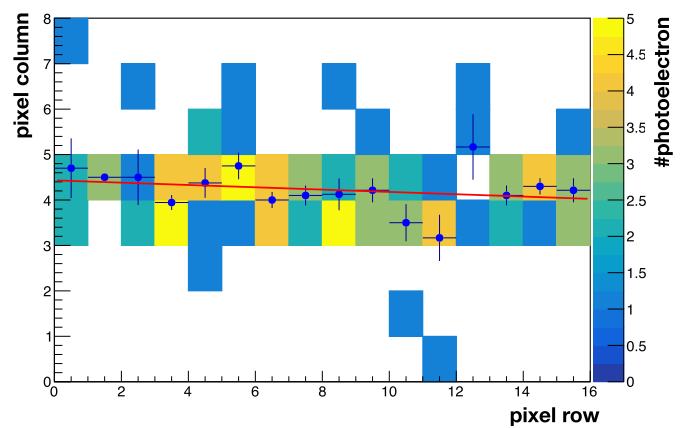

Fig. 11. Example of a $140 \mathrm{MeV}$ proton track reconstructed by the SPAD-net sensor. The beam is coming from left to right.
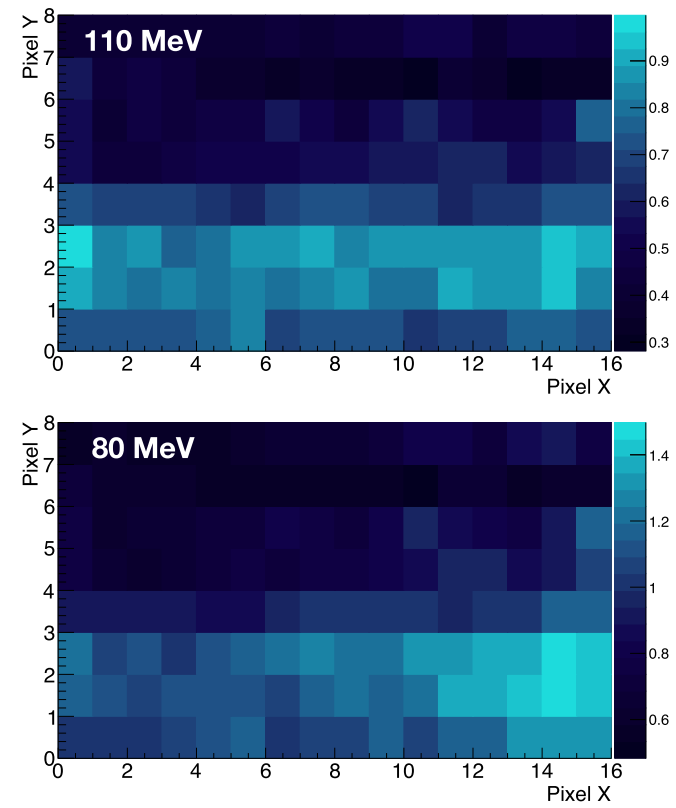

Fig. 12. Map of the sensor, showing the average number of photoelectrons per pixel measured for impinging protons of $110 \mathrm{MeV}$ (top) and $80 \mathrm{MeV}$ (bottom) energy. The beam is directed from left to the right.

been readout (see Fig. 10). The sensor has been positioned in order to observe the tracks of the softest protons that stop inside the tracker prototype. A top view scheme of Penelope and the readout is shown in Fig. 10. The studies of the tracking reconstruction capability and of the energy release are presented here.

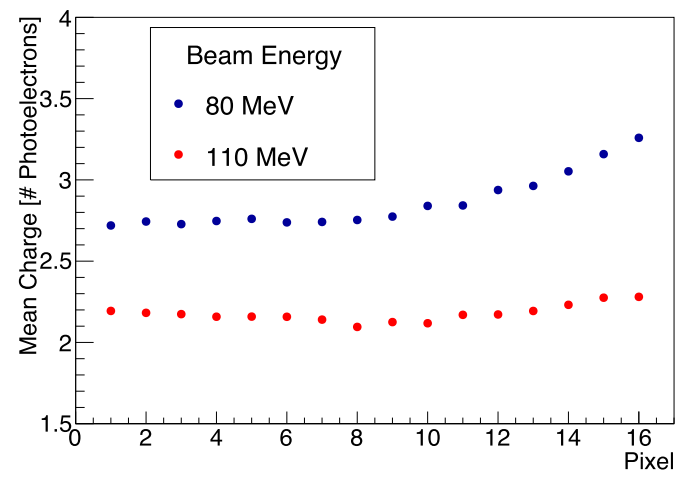

Fig. 13. Mean charge collected in function of the pixel $x$ value for 80 and $110 \mathrm{MeV}$. The values reported in the plot are obtained from the sum of the average photoelectron contained in a column of pixel.

An example of a $140 \mathrm{MeV}$ proton track reconstructed by the SPAD-net sensor is shown in Fig. 11, where the map of pixel from the row and columns of the SPAD-net matrix is shown. In Fig. 12, the sensor map, averaged over 75000 events, is shown for two different beam energies (110 and $80 \mathrm{MeV}$ ). The brighter band in the bottom side of the plots is related to the proton traversing the detector. As the beam proceeds along the $x$-axis, the pixel counts increase, as expected for a proton that is slowing down. Since the energy loss along the proton track can be potentially exploited in order to obtain information on its kinetic energy, a study of the deposited energy as a function of the proton track has been performed. In Fig. 13, the mean charge deposit per pixel is shown. Charge is reported in terms of the number of photoelectrons, and corresponds to the deposited energy collected by the pixel row (pixel y) as a function of the pixel column (pixel $\mathrm{x}$ ). For the $80 \mathrm{MeV}$ distribution, the $\mathrm{BP}$ is in correspondence of the last two pixel $\mathrm{x}$. It can be noticed that the deposited energy (charge collected) by pixels for protons of $80 \mathrm{MeV}$ kinetic energy is greater than that in the $110 \mathrm{MeV}$ case. This is a first hint for the possible exploit of the energy loss measurements in proton kinetic energy evaluation, thus, the possibility to release the full containment condition in the neutron DES reconstruction.

\section{CONCLUSion}

The DP and the MONDO tracker are two detectors that have been developed for light ion $\left({ }^{4} \mathrm{He},{ }^{12} \mathrm{C},{ }^{16} \mathrm{O} \ldots\right)$ PT application purposes. In particular, the DP aim is to work as an online beam range monitor exploiting the charged secondary radiation (mainly secondary protons) that exits the patient body. The MONDO detector has been developed in order to characterize the secondary fast and ultrafast neutron production in PT treatments. Such frontier study would allow for an accurate and complete description of the neutron effects on tissues, especially in patients with long survival expectation (in particular pediatric patients), helping to improve the treatment plans accuracy.

The DP and the MONDO trackers are based on plastic scintillating fibers and have been designed in order to be economic, compact and, therefore, easily integrable in a PT treatment room. First, results have been reported for the data 
taking performed in May 2017 at the Trento Protontherapy Center in order to test the performances of the DP and the MONDO tracker prototype. The obtained results have shown that a spatial resolution on a single reconstructed proton track of the order of 3-5 mm can be achieved with the DP: the measured spatial resolution on the fragment emission point is consistent with the expectations and will allow to match the precision $(\sim$ millimeter $)$ required in $\mathrm{PT}$ online beam range monitoring applications, once the suitable charged particle statistics are collected.

Within the MONDO project, the measurements performed at the Trento Protontherapy Center, using Penelope and the SPAD-net readout prototype, were intended to study the feasibility of the SPAD technology application to the final MONDO tracker readout: the SBAM sensor, currently in development in collaboration with FBK. The first test chips of SBAM will be available before the end of 2018 .

\section{ACKNOWLEDGMENT}

The authors would like to thank TIFPA, the Trento Protontherapy Center, and the Centro Nazionale di Adroterapia Oncologica staff for beam time and helpful support during the data taking.

\section{REFERENCES}

[1] O. Jäkel, C. P. Karger, and J. Debus, "The future of heavy ion radiotherapy," Med. Phys., vol. 35, no. 12, pp. 5653-5663, 2008.

[2] M. Durante and J. S. Loeffler, "Charged particles in radiation oncology," Nature Rev. Clin. Oncol., vol. 7, pp. 37-43, Dec. 2010.

[3] F. Tommasino, E. Scifoni, and M. Durante, "New ions for therapy," Int. J. Part. Therapy, vol. 2, no. 3, pp. 428-438, 2015.

[4] H. Paganetti, "Range uncertainties in proton therapy and the role of Monte Carlo simulations," Phys. Med. Biol., vol. 57, no. 11, pp. R99-R117, 2012.

[5] V. Batista, D. Richter, S. E. Combs, and O. Jäkel, "Planning strategies for inter-fractional robustness in pancreatic patients treated with scanned carbon therapy," Radiat. Oncol., vol. 12, p. 94, Jun. 2017.

[6] A.-C. Knopf and A. Lomax, "In vivo proton range verification: A review," Phys. Med. Biol., vol. 58, no. 15, pp. 131-160, 2013.

[7] I. Mattei et al., "Prompt- $\gamma$ production of $220 \mathrm{MeV} / \mathrm{u}{ }^{12} \mathrm{C}$ ions interacting with a PMMA target," J. Instrum., vol. 10, p. P10034, Oct. 2015.

[8] M. Pinto et al., "Absolute prompt-gamma yield measurements for ion beam therapy monitoring," Phys. Med. Biol., vol. 60, pp. 565-594, Jan. 2015.
[9] I. Mattei et al., "Secondary radiation measurements for particle therapy applications: Prompt photons produced by ${ }^{4} \mathrm{He},{ }^{12} \mathrm{C}$ and ${ }^{16} \mathrm{O}$ ion beams in a PMMA target," Phys. Med. Biol., vol. 62, no. 4, pp. 1438-1455, 2017.

[10] W. Enghardt et al., "Charged hadron tumour therapy monitoring by means of PET," Nucl. Instrum. Methods Phys. Res. A, Accel. Spectrom. Detect. Assoc. Equip., vol. 525, pp. 284-288, Jun. 2004.

[11] C. Agodi et al., "Study of the time and space distribution of the $\beta^{+}$ emitters from $80 \mathrm{MeV} / \mathrm{u}$ carbon ion beam irradiation on PMMA," $\mathrm{Nucl}$. Instrum. Methods Phys. Res. B, Beam Interact. Mater. At., vol. 283, pp. 1-8, Jul. 2012.

[12] L. Piersanti et al., "Measurement of charged particle yields from PMMA irradiated by a $220 \mathrm{MeV} / \mathrm{u}{ }^{(12)} \mathrm{C}$ beam," Phys. Med. Biol., vol. 59, no. 7, p. 1857,2014

[13] K. Gwosch et al., "Non-invasive monitoring of therapeutic carbon ion beams in a homogeneous phantom by tracking of secondary ions," Phys. Med. Biol., vol. 58, no. 11, p. 3755, 2013.

[14] J. Smeets et al., "Prompt gamma imaging with a slit camera for realtime range control in proton therapy," Phys. Med. Biol., vol. 57, no. 11, pp. 3371-3405, 2012

[15] C. Richter et al., "First clinical application of a prompt gamma based in vivo proton range verification system," Radiotherapy Oncol., vol. 118, no. 2, pp. 232-237, 2016

[16] M. A. Piliero et al., "First results of the INSIDE in-beam PET scanner for the on-line monitoring of particle therapy treatments," J. Instrum., vol. 11, p. C12011, Dec. 2016.

[17] M. G. Bisogni et al., "INSIDE in-beam positron emission tomography system for particle range monitoring in hadrontherapy," J. Med. Imag., vol. 4, no. 1, p. 011005,2016

[18] G. Traini et al., "Design of a new tracking device for on-line beam range monitor in carbon therapy," Phys. Medica, vol. 34, pp. 18-27, Feb. 2017.

[19] W. D. Newhauser and M. Durante, "Assessing the risk of second malignancies after modern radiotherapy," Nature Rev. Cancer, vol. 11, no. 6, pp. 438-448, Jun. 2011.

[20] M. Marafini et al., "MONDO: A neutron tracker for particle therapy secondary emission characterisation," Phys. Med. Biol., vol. 62, no. 8 , pp. 3299-3312, 2017, doi: 10.1088/1361-6560/aa623a.

[21] K. Gunzert-Marx, H. Iwase, D. Schardt, and R. S. Simon, "Secondary beam fragments produced by $200 \mathrm{MeV} \mathrm{u}^{-1}{ }^{12} \mathrm{C}$ ions in water and their dose contributions in carbon ion radiotherapy," New J. Phys., vol. 10, p. 075003 , Jul. 2008

[22] F. Ciciriello et al., "BASIC32 ADC, a front-end ASIC for SiPM detectors," in Proc. IEEE NSS/MIC, Oct./Nov. 2013, pp. 1-6.

[23] A. Ferrari, P. R. Sala, A. Fasso, and J. Ranft, "FLUKA: A multi particle transport code," Tech. Rep. CERN-2005-010, INFN TC 05/11, SLAC-R-773, Oct. 2015

[24] G. Battistoni et al., "Overview of the FLUKA code," Ann. Nucl. Energy, vol. 82, pp. 10-18, Aug. 2015.

[25] L. H. C. Braga et al., "A fully digital $8 \times 16 \mathrm{SiPM}$ array for PET applications with per-pixel TDCs and real-time energy output," IEEE J. Solid-State Circuits, vol. 49, no. 1, pp. 301-314, Jan. 2014.

[26] R. Mirabelli et al., "The MONDO detector prototype development and test: Steps toward an SPAD-CMOS-based integrated readout (SBAM sensor)," IEEE Trans. Nucl. Sci., vol. 65, no. 2, pp. 744-751, Feb. 2018. 